Supplement Issue 1: International Tehran Breast Cancer Congress (TBCC9)

\title{
Exploring the Problems and Concerns of Spouses of Patients with Breast Cancer: A Qualitative Study
}

\author{
A Sajadian ${ }^{1,}$, , A Motaharinasab ${ }^{1}$, L M Raji ${ }^{1}$, B Chegini ${ }^{1}$ \\ ${ }^{1}$ Quality Of Life Department, Breast Cancer Research Center, Motamed Cancer \\ Institute, Tehran, Iran \\ ${ }^{*}$ Corresponding author: A Sajadian, Quality Of Life Department, Breast Cancer \\ Research Center, Motamed Cancer Institute, Tehran, Iran.E-mail: assajadi@yahoo.com
}

DOI: $10.21859 /$ mci-supp-97

\section{Keywords:}

Breast Cancer

Qualitative Study

Spouses' Problem

Iran

\begin{abstract}
Introduction: Women have a special position in family structures and breast cancer diagnosis has a negative effect on patient's spouse. The personal, familial and sexual functioning disturbances are most common among breast cancer spouses. The aim of this qualitative study was to explore the spouses' problems after their women breast cancer diagnosis, in Iran.

Materials and Methods: Twenty three participants were recruited from a university-affiliated breast clinic in Tehran. In-depth interviews each lasting approximately one hour per participant were scheduled and conducted in a private room. The interviews intended to motivate the participants to reflect on their problems since their women's breast cancer diagnosis. The interviews were recorded and were transcribed to elucidate the major themes encountered in the interviews.

Results: The mean age of spouses was 50/7, (SD =6/97), 19 participants ( $82 \%)$ were up to diploma educated, and also 12 person (52\%) were self employee. Overall, two major them were emerged from the analysis these were: 1 ) physical and psychological problems (sexual, fatigue, anger, feeling of guilt, sadness). 2) Concerns (worries about metastasizing and about their health and death and their wives fear of disclosure, bad event, financial problem).

Conclusions: This study showed the main problems of patient's spouses were fatigue, feel guilty, anger, and lack of control and fear of metastasis. These mental and physical problems are important for patient's continuing treatment. Therefore, recommended performing beneficial interventions to support patient's families to improving quality of life.
\end{abstract}

